\title{
Unobtrusive Health Screening on an Intelligent Toilet Seat
}

\author{
T. Schlebusch
}

\begin{abstract}
Home monitoring is a promising way to improve the quality of medical care in an ageing society. To circumvent the problem that especially demented patients may forget or be stressed by the use of medical devices at home, monitoring devices should be embedded in objects of daily life to check the patient's health status whenever possible, without any interaction with the patient him/herself. This paper presents an intelligent toilet performing an unobtrusive health check when a person sits down. A variety of physical, electro-physical and urine parameters are analysed. This paper takes electrocardiogram and bioimpedance spectroscopy measurements and shows the practicability of measuring them on a toilet seat.
\end{abstract}

Keywords: monitoring of vital parameters, personal healthcare, ambient assisted living, electrocardiogram, bioimpedance, toilet seat.

\section{Introduction}

German society is undergoing a profound change in its age distribution. In about thirty years, around one third or the German population will be over 65 years old. Along with the change in age distribution comes also a change in the main disease patterns. Chronic diseases such as diabetes and chronic heart failure will further gain in relevance and, if not detected at an early stage, these diseases will burden the health systems with overwhelming costs. To limit public health expenditure and ensure appropriate health care and quality of life for patients, automatic monitoring of vital functions at home is an important building block for future health care systems.

Commercial home monitoring systems such as the "Telemedizin für's Herz" programme of the German health insurance company $\mathrm{TK}^{1}$ rely on interaction between a patient with a device and a telemedicine center. This means the patient him/herself has to take measurements with several devices, for example a weighing scale, a blood pressure monitor and probably even more devices, and note the measured values. The patient then calls the telemedicine center and transmits the measured parameters by phone. This leads to a very high daily workload for the patient if the number of monitored parameters increases. Further, the scheme is unfeasible for elderly patients, who may have problems controlling the measurement devices or may forget to take the measurements.

To overcome these drawbacks, more and more research projects have tried to take the burden of daily measurements from the patient and perform them unobtrusively during normal daily activities. One solution is to embed the measurement electronics in textiles worn by the patient, e.g. an ECG shirt [1] or to measure the body composition by electronics integrated into smart clothing [2]. These systems provide the best long-term monitoring of patients, but can not be treated like regular clothing: the batteries need to be charged regularly, and the electronics has to be removed before washing. The second approach is to embed monitoring electronics into devices used by the patient in his daily life, e.g. sleep-monitoring in his bed $[3,4]$ or monitoring of the heart function on his chair [5], in his bath tub [6] or on his toilet seat [7-9]. While measurements in a bed or on a chair always have to deal with the clothing between the capacitive sensor and the human body, measurements on a toilet seat provide direct skin contact to the sensors and even enable urine analysis [10].

For early detection of a disease, e.g. diabetes or chronic heart failure, more than one parameter has to be measured and tracked for parameter trend analysis. In the following sections an intelligent toilet will be presented that measures a wide variety of vital parameters, see Figure 1. The intelligent toilet detects that a user sits down by regularly polling the weight sensors in the toilet seat. When a person sits down, automatic measurement is started and the results are transmitted wirelessly to a central control unit (CCU), as shown in Figure 2. The CCU can collect data from several intelligent toilets, e.g. from different apartments in a retirement home, and forward them via a mobile phone or an internet connection to a central database. The database holds records for each patient (identified by toilet-ID) and performs several threshold operations on the vital parameters and their calculated trends. In this way for

\footnotetext{
${ }^{1}$ http://www.tk.de/tk/innovative-verfahren/telemedizin/herz/9784
} 
example not only a threshold for the absolute weight of a patient can be monitored, but also a threshold for weight loss per time can be set.

This paper will emphasize two important technological aspects of the intelligent toilet and will focus in detail on electrocardiogram (ECG) and on bioimpedance spectroscopy (BIS) analysis.

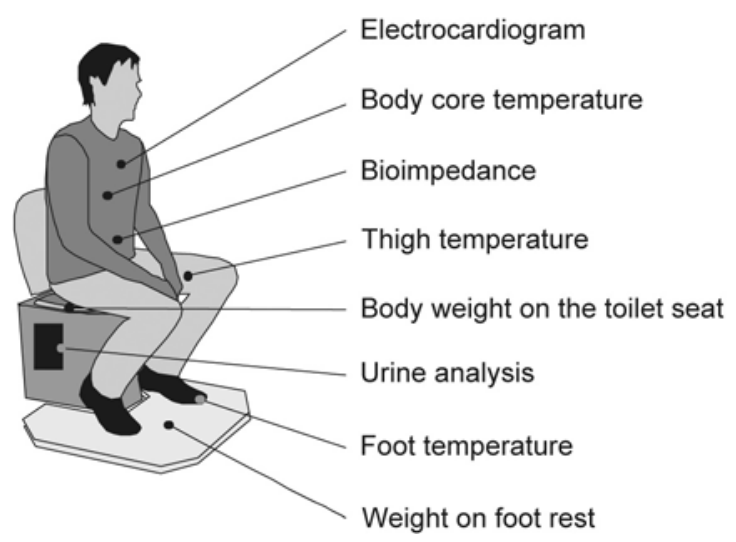

Fig. 1: Parameters measured by the intelligent toilet

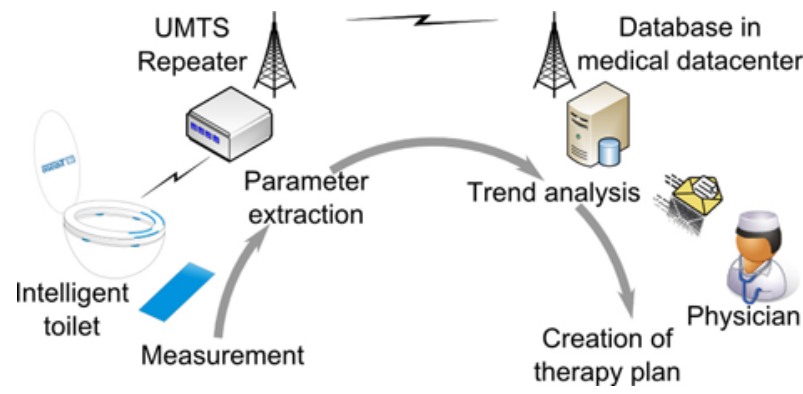

Fig. 2: Overview of the data transmission setting from the intelligent toilet to the physician

\section{Theoretical background}

An electrocardiogram represents the potential caused by the electric activity of the heart measured on the body surface over time. Since the cardiac pulse spreads from the right atrium over the heart, the resulting electrical potential can be measured between two opposite places on the human chest. The resulting field and equipotential lines are shown in principle in Figure 3. The strength of the recorded ECG signal is dependent on the strength of the potential difference between the electrode positions. From two opposite positions on the chest, positions A and B in Figure 3, a higher amplitude can be measured than between positions $\mathrm{C}$ and $\mathrm{D}$, as we have for the ECG measurement on a toilet seat. Since the amplitude is several orders smaller than the conventional chest ECG, most commercial ECG recorders will not be able to record any signal. For the measurements on a toilet seat, special hardware with cascaded gain and filter stages with an overall gain of 12000 has been constructed.

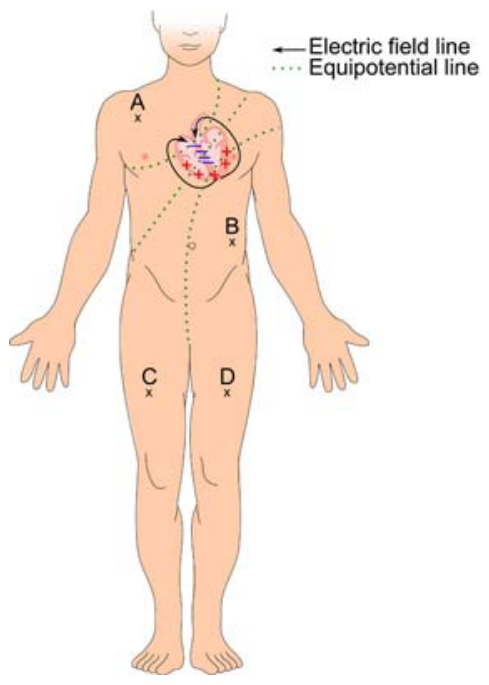

Fig. 3: Equipotential lines on the surface of a human body, data for the simplified illustration taken from [11, $12]$

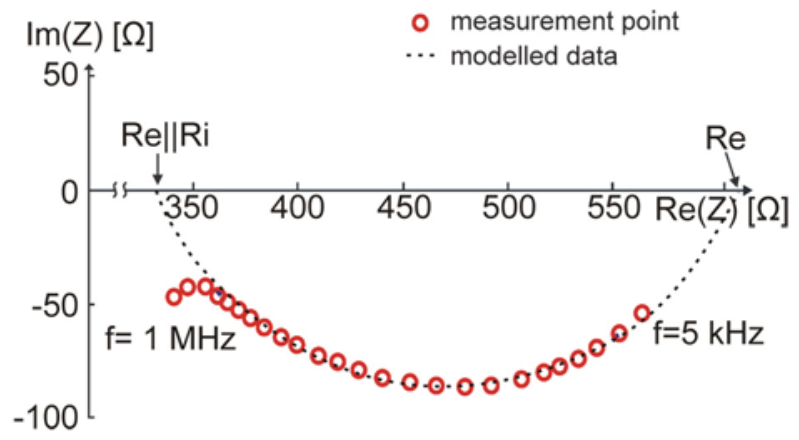

Fig. 4: Typical BIS measurement raw data

Bioimpedance Spectroscopy is a method for determining body composition [14] by measuring the complex impedance of tissue over a wide frequency range, usually in the range from $5 \mathrm{kHz}$ to $1 \mathrm{MHz}$ [13]. A very low alternating current $i(j \omega)$ is injected by two current electrodes applied to the human body. Between the current electrodes, two additional voltage electrodes are placed that measure the resulting voltage drop $u(j \omega)$. The complex impedance can then be reconstructed by $Z(j \omega)=u(j \omega) / i(j \omega)$ for each frequency. By using separate electrodes for current injection and for voltage measurement, the effect of the electrode-skin contact impedance can be neglected. A typical plot of the acquired impedance data in the complex impedance plane is shown in Figure 4. Cole [15] has shown that cell membranes show capacitive behaviour. At DC level the measurement current cannot pass the cell membrane and can flow only in the extracellular space. Towards higher fre- 
quencies, the current can pass the cell membrane and take a shorter path through the cells. This can be modelled by an equivalent model having resistance $R_{e}$, denoting extracellular resistance, in parallel to the series connection of capacitor $C_{m}$ and resistance $R_{i}$, denoting the capacitance of the cell membranes and the intracellular resistance, respectively.

For determining the hydration status of a person, $R_{e}$ is of great interest, since a tight correlation between total body water and a change in $R_{e}$ has been shown [16]. The BIS electrodes are usually applied to the right hand and the right foot, forming a whole body measurement. Medrano [16] was recently able to show that a measurement between the legs, as in our toilet-seat setting, could be sufficient for reliably determining the amount of body fluid.

\section{Materials and Methods}

Before being able to take first measurements with the intelligent toilet, it was necessary to embed adequate electrodes with the toilet seat. Four electrodes are necessary for BIS measurements, and it was decided to reuse them to connect the three ECG cables. For ECG and BIS measurements, hydro-gel electrodes are usually used. They consist of metallic electrodes such as silver/silver-chloride or aluminium with a layer of hydro-gel, which is also used as a glue layer to attach the electrodes to the skin. These electrodes have a number of advantages, but unfortunately they are disposables. This prevents their use in a maintenance-free toilet seat measurement system. In search of appropriate electrodes for integration in a toilet seat, it was decided to use printed circuit boards (PCB) as the basic material, since they are cheap and easy to process. A layer of tin was applied chemically to the copper layer of the PCB, and then a gold layer was applied by a galvanic process. The final electrode showed good contact impedances and long-term stability. The gold layer makes the electrode surface biocompatible, which is important for direct contact with human skin.

Since the electrodes have to be placed on the toilet seat in such a way that all individuals are in good contact with them, a toilet seat was equipped with pressure sensors and ten student volunteers (seven male and three female) were asked to sit as they usually do when they use a toilet. The area featuring the highest contact pressure for all individuals was then chosen as the right place to embed the electrodes on the toilet seat.

The ECG measurement was made by connecting the developed ECG-amplifier to the toilet seat electrodes. As a reference, a conventional lead-1 chestECG was taken with a Bsamp biosignal amplifier (g.tek medical engineering GmbH, Schiedlberg, Austria). Normal hydro-gel electrodes were used for the reference ECG. The outputs of both amplifiers were connected to a National Instruments USB data acquisition card, enabling synchronous recording with the use of a connected laptop. Unfortunately, the driven right leg lead of the Bsamp amplifier could not be connected with the subject since it interfered with the toilet seat ECG amplifier, resulting in a higher noise level on the reference ECG than is usually present.

For BIS measurements, the ECG electronics was disconnected from the toilet seat and a Hydra 4200 (Xitron Technologies, USA) BIS device was connected. It was also connected to a PC by a serial connection, and a LabView programme was used to record the raw measurements from the BIS device. After taking a measurement on the toilet seat, aluminium hydro-gel electrodes (Fresenius Medical Care, Bad Homburg, Germany) were attached to the legs of the subject. An effort was made to attach them as well as possible to the same place where the toilet seat electrodes also connected to the legs.

\section{Results}

\subsection{Electrode positioning}

The pressure measurements on the toilet seat showed interestingly clear differences between genders. Representative plots of the weight distribution for all ten measurements on the toilet seat are shown for a male subject in Figure 7a) and for a female subject in Figure $7 \mathrm{~b}$ ). Fortunately, though the pressure distributions are so different for the two genders, the areas of highest contact pressure (red or orange in Figure 7a) and $7 \mathrm{~b})$ ) are in the same region. The four electrodes were then embedded in this region, see Figure 5.

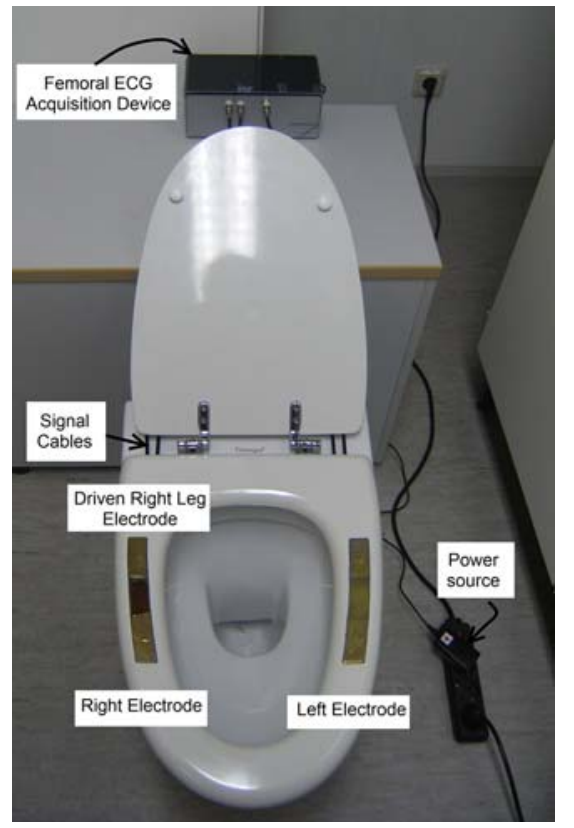

Fig. 5: Picture of the experimental setup in the lab 

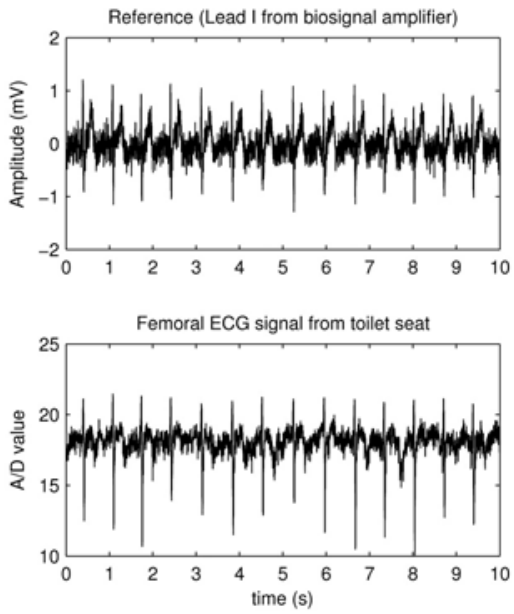

Fig. 6: Comparison of toilet seat ECG with conventional lead 1

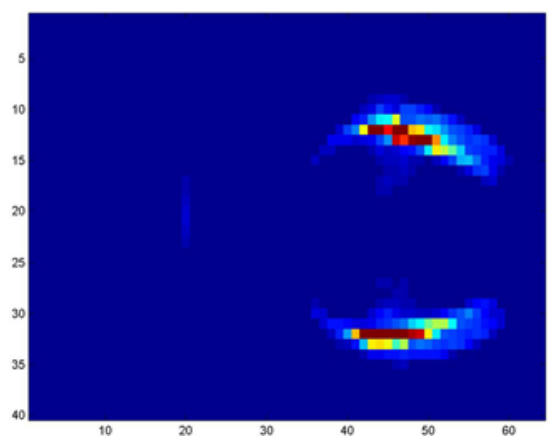

a) typical male

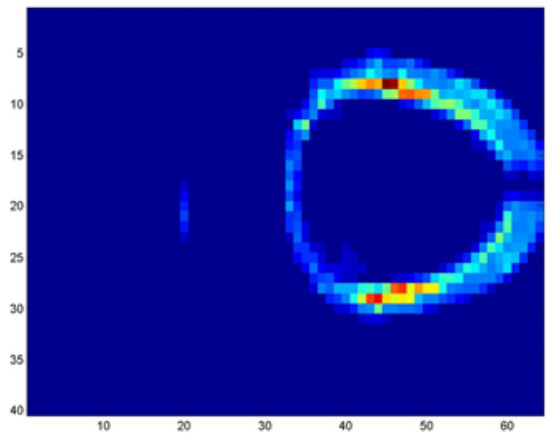

b) typical female

Fig. 7: Weight distribution on a toilet seat

\subsection{ECG measurement}

An example of an ECG measurement is shown in Figure 6 . The upper diagram shows the raw data from the data acquisition card for the reference ECG, while the lower diagram shows the raw data from the toilet seat ECG amplifier. As mentioned above, it was not possible to connect the driven right leg electrode of the biosignal amplifier to the subject since it would interfere with the toilet seat electronics. It was only possible to connect one of the two active feedback electrodes, so the feedback from the toilet seat ECG was chosen. This results in a much higher noise floor for the reference ECG.

In the toilet seat ECG the R-peak is clearly visible, enabling R-peak-detection and an estimation of heart rate and heart rate variability. It is hardly possible to detect additional information, e. g. propagation delays, without further signal processing. The intelligent toilet is equipped with an MSP430F5437A microprocessor (Texas Instruments, USA), and the EP Limited Open Source ECG Analysis Software ${ }^{2}$ was ported to this processor. By connecting the toilet seat ECG amplifier to the integrated A/D converter of the microprocessor it was possible to automatically analyse the heart rate of a subject on the toilet seat. The system was tested for its robustness against motion artefacts. Since the author expects motion on the toilet seat usually to be limited to leaning forwards or sidewards, only these movements were tested. A reliable measurement could be made during most movements. Only when leaning extensively sidewards could the connection between one leg and the electrodes get lost, thus interrupting the ECG signal.

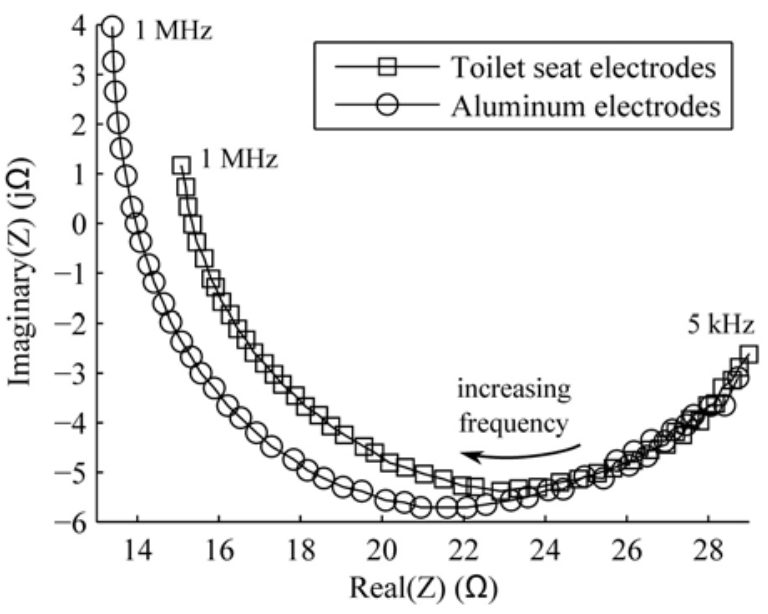

Fig. 8: Comparison of a BIS measurement using the dry electrodes in the toilet seat and one reference measurement using aluminium hydro-gel electrodes

\subsection{BIS measurement}

BIS measurements could be taken using the dry electrodes embedded in the toilet set. Figure 8 shows both the toilet seat and the reference measurements. For low frequencies starting from $5 \mathrm{kHz}$ both measurement methods yield the same results. For higher frequencies, especially the reference measurement using aluminium hydro-gel electrodes reveals results with a positive imaginary part, which can be qualified

\footnotetext{
${ }^{2}$ http://www.eplimited.com, last checked 8/3/2011
} 
as a measurement error. As has also been observed in other measurement scenarios using the Hydra 4200, use of the device in other impedance ranges than whole body measurements leads to problems with the current source of the device. The device is intended for whole body measurements at resistances around $900 \Omega$. In our measurement setup, the impedance is several orders lower, clearly leading to measurement errors for higher frequencies. For accurate results in the whole frequency range, the development of a special BIS device for the toilet seat BIS measurement would be necessary. Nevertheless, the results are of medical value: even with errors in the impedance data for high frequencies the Cole-Cole parameter $R_{e}$ could be extracted. As mentioned above, this parameter is of great interest since it correlates with the hydration status of the patient.

\section{Conclusions}

An intelligent toilet has been constructed which can perform a comprehensive health check when a person sits down. This paper has shown the practicability of ECG and BIS measurements using dry electrodes embedded in a toilet seat. A biosignal amplifier with an overall gain of 12000 has been constructed for measuring the ECG signal. It has been shown that the signal is good enough to perform reliable R-peak detection, which can be used to estimate the heart rate and heart rate variability. Typical light movements on the toilet seat are extraneous to the measurement. Monitoring body composition using BIS has also been shown to be possible using the same electrodes. The intelligent toilet presented here has high potential to increase the quality of medical care for elderly people living at home by keeping track of important health and nutrition parameters. When a deviation of the parameters is detected, a medical professional can be informed to get in contact with the patient.

\section{Acknowledgement}

Research presented in this paper was supervised by Univ.-Prof. Dr.-Ing. Dr. med. S. Leonhardt, Chair for Medical Information Technology at RWTH Aachen, and has been supported by the Federal Ministry of Economics and Technology (BMWi) and the German Federation of Industrial Research Associations (AiF) under grant No. KF2561903FO9.

This study forms part of a joint research programme with our partners Kurt-Schwabe-Institut für Mess- und Sensortechnik e. V. Meinsberg, ClinPath $\mathrm{GmbH}$ Berlin, Innotas Elektronik GmbH Zittau and BitsZ Engineering Zwickau.

\section{References}

[1] Lee, Y.-D., Chung, W.-Y.: Wireless sensor network based wearable smart shirt for ubiquitous health and activity monitoring, Sensors and Actuators B, vol. 140, 2009, pp. 390-395.

[2] Vuorela, T., Kukkonen, K., Rantanen, J., Järvinen, T., Vanhala, J.: Bioimpedance Measurement System for Smart Clothing, Proceedings of the Seventh IEEE International Symposium on Wearable Computers ISWC'03, 2003.

[3] Zhu, X., Chen, W., Nemoto, T., Kanemitsu, Y., Kitamura, K., Yamakoshi, K., Wei, D.: RealTime Monitoring of Respiration Rhythm and Pulse Rate During Sleep, IEEE Transactions on Biomedical Engineering, vol. 53, 2006, pp. 2553-2 563 .

[4] Watanabe, K., Watanabe, T., Watanabe, H., Ando, H., Iskikawa, T., Kobayashi, K.: Noninvasive Measurement of Heartbeat, Respiration, Snoring and Body Movements of a Subject in Bed via a Pneumatic Method, IEEE Transactions on Biomedical Engineering, vol. 52, 2005, pp. 2100-2 107.

[5] Aleksandrowicz, A., Walter, M., Leonhardt, S.: Wireless ECG measurement system with capacitive coupling, Biomedizinische Technik, vol. 52, Nr. 2, 2007, pp. 185-192.

[6] Lim, Y. K., Kim, K. K., Park, K. S.: The ECG measurement in the bathtub using the insulated electrodes, Engineering in Medicine and Biology Society, IEMBS '04. 26th Annual International Conference of the IEEE, 2004, pp. 2 383-2385.

[7] Kim, K. K., Lim, Y. K., Park, K. S.: The Electrically Non-contacting ECG Measurement on the Toilet Seat Using the Capacitively-coupled Insulated Electrodes, Proceedings of the 26th Annual International Conference of the IEEE EMBS, 2004, pp. 2375-2378.

[8] Baek, H. J., Kim, J. S., Kim, K. K., Park, K. S.: System for Unconstrained ECG Measurement on a Toilet Seat using Capacitive Coupled Electrodes: The Efficacy and Practicality, 30th Annual International IEEE EMBS Conference, 2008, pp. $2326-2328$.

[9] Kim, J. S., Chee, Y. J., Park, J. W., Choi, J. W., Park, K. S.: A new approach for nonintrusive monitoring of blood pressure on a toilet seat, Physiological Measurement, vol. 27, 2006, pp. 203-211.

[10] Fichtner, W., Schlebusch, T., Leonhardt, S., Mertig, M.: Photometrische Urinanalyse als 
Baustein für ein mobiles Patientenmonitoring mit der Intelligenten Toilette, 3. Dresdner Medizintechnik-Symposium, Dresden, Dec. 6th-9th, 2010.

[11] Sachse, F. B., Werner, C. D., Meyer-Waarden, K., Dössel, O.: Applications of the Visible Man Dataset in Electrocardiology: Calculation and Visualization of Body Surface Potential Maps of a Complete Heart Cycle, Second Users Conference of the National Library of Medicine, 1998, pp. S. 47-48.

[12] Schneider, F., Dössel, O., Müller, M.: Optimierung von Elektrodenpositionen zur Lösung des inversen Problems der Elektrokardiographie, Biomedizinische Technik, vol. 43, 1998, pp. $58-59$.

[13] Moissl, U., Wabel, P., Leonhardt, S., Isermann, R.: Anwendungen-Modellbasierte Analyse von Bioimpedanz-Verfahren, Automatisierungstechnik, vol. 52, nr. 6, 2004, pp. 270-279.

[14] Kyle, U. G., Bosaeus, I., De Lorenzo, A. D., Deurenberg, P., Elia, M., Gomez, J. M., Heitmann, B. L., Kent-Smith, L., Melchior, J. C., Pirlich, M. et al.: Bioelectrical impedance analysis - part I: review of principles and meth- ods, Clinical Nutrition, vol. 23, nr. 5, 2004, pp. $1226-1243$.

[15] Cole, K. S.: Membranes, ions, and impulses. Berkeley : University of California Press, 1968.

[16] Medrano, G., Eitner, F., Floege, J., Leonhardt, S.: A Novel Bioimpedance Technique to Monitor Fluid Volume State During Hemodialysis Treatment, ASAIO Journal, vol. 56, nr. 3, 2010, pp. 215-220.

\section{About the author}

Thomas Schlebusch studied Electrical Engineering at RWTH Aachen University, Germany, and at NTNU Trondheim University, Norway. He is now a Ph.D. student with the Institue for Medical Information Technology at RWTH Aachen, Germany. His current research fields are home monitoring, textile integration and impedance spectroscopy.

Thomas Schlebusch

E-mail: schlebusch@hia.rwth-aachen.de

Philips Chair for Medical Information Technology

Helmholtz Institute for Biomedical Engineering RWTH Aachen, Pauwelsstr. 20, 52074 Aachen, Germany 